
2 Research Square
Preprints are preliminary reports that have not undergone peer review.
They should not be considered conclusive, used to inform clinical practice,
or referenced by the media as validated information.

\title{
The effect of quitting water pipe during pregnancy on Anthropometric measurements at birth: a population-based prospective cohort study in South of Iran
}

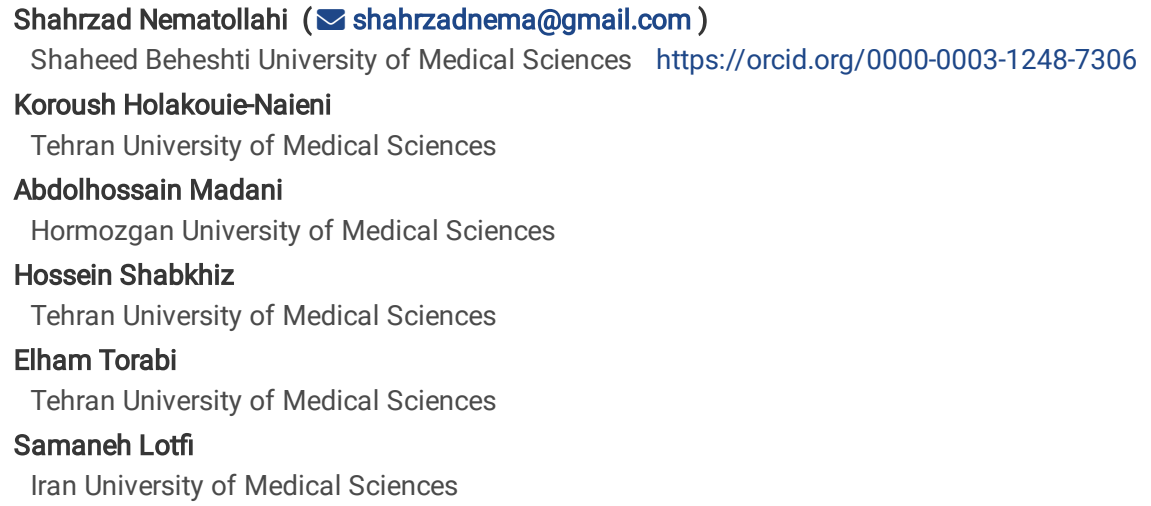

Version of Record: A version of this preprint was published at BMC Pregnancy and Childbirth on April 22nd, 2020. See the published version at https://doi.org/10.1186/s12884-020-02948-2. 


\section{Abstract}

Background Evidence on health effects of tobacco cessation in pregnancy is mainly restricted to cigarette, while water pipe smoking is more prevalent among women in the Middle-East. The present study aimed to assess the effects of cessation of water pipe during pregnancy on birth anthropometric measures in South of Iran. Methods Data on 1,120 singleton pregnancies (response rate=93.4\%) from a population-based prospective cohort study in suburban communities in Bandar Abbas city was used. Birth measures were used according to infant's vaccination cards. Study subjects were categorized into three subgroups based on water pipe smoking status during pregnancy, including: 1) mothers who never smoke water pipe (never smoker); 2)mothers who stopped water pipe during pregnancy and resumed it postpartum (quitters); 3 ) mothers who continued smoking water pipe regardless of their pregnancy (always smokers). Generalized Linear Models (GLMs) were utilized for the analyses. Results Compared to never smokers, quitting water pipe in pregnancy would decrease mean birthweight by 99.30 grams ( $\beta$ :-99.30, 95\%Cl:-204.35,-5.75); while an additional decrease by 37.83 grams would occur in always smokers ( $\beta:-137.13 ; 95 \% \mathrm{Cl}:-262.21,-12.05)$. Mean birth length did not significantly differ among the three water pipe groups. Mean head circumference, however, would significantly increase by 0.79 centimeter in always smokers ( $\beta: 079,95 \% \mathrm{Cl}: 0.13,1.45)$. Conclusion Quitting water pipe during pregnancy had positive effects on infant growth, especially birth weight. Some suggestions are provided including integration of information on health benefits of quitting smoking into routine prenatal healthcare services and development of awareness programs to encourage smokers to quit early in pregnancy.

\section{Background}

Due to the well-established adverse effects of cigarette smoking on pregnancy outcomes (1), attention now has been shifted to other types of tobacco use, such as water-pipe smoking. The tradition of water pipe (also known as narghile, hookah, and shisha) dates back 400 years in Turkey, India, and Iran (2), and in spite of deleterious health effects, it is widely believed to be a less harmful form of tobacco consumption, and a safer alternative to cigarette smoking (3). In the south of Iran, reports have shown that approximately 8.0-14.0\% of pregnant women use water pipe regularly $(4,5)$. The adverse effects of water pipe on fetal growth have been the focus of very limited research in Iran. Studies reported that the probability of intrauterine growth retardation increased by 2-3.5 times in water pipe smokers in Bandar Abbas $(6,7)$. On the other hand, a negative correlation has been reported between infant's mean birth weight and extent of maternal cigarette smoking (8). The promising results of smoking cessation during gestational period on infant growth and pregnancy outcomes have been reported in few studies, mostly from developed countries $(9,10)$. At the same time, water pipe has been gaining popularity among Iranian women, which necessitates investigation on its health effects with special consideration to reproductive outcomes. To the best of our knowledge; however, there is no epidemiologic evidence on the effects of water pipe cessation on fetal growth among Iranian population. Such epidemiologic evidence is required to recommend quit smoking during pregnancy period; while piled-up recommendations can be integrated into prenatal healthcare services. Therefore, the aim of the present study was to estimate the effects of water pipe cessation during pregnancy on birth anthropometric measures in South of Iran.

\section{Methods}

We used data from a prospective cohort study entitled "A population-based prospective cohort study to identify contributors of mother and child health in suburban communities" in Bandar Abbas city, which is abbreviated as Bandar Abbas Pregnancy Cohort (BAPC). BAPC aims to determine the magnitude of effects of lifestyle and environmental factors on maternal and child health among residents of suburban neighborhoods of Bandar Abbas, an unindustrialized region in the South of Iran. Subjects were recruited through home-by-home sampling scheme. All pregnant women above the age of 16 who were residing in the study area at least 6 months prior to the study were eligible to participate. Exclusion criteria was defined for those who had medically-assisted conception or were unwilling to participate. Follow-up visits were performed at home by trained interviewers. The study protocol is ethically approved and financially funded by the National Institute for Medical Research Development (with the approval code: 943607 and ethical code: N. IR.NIMAD.REC.1396.205). The details of the methodology have been published elsewhere (11). The present study used data on 1,120 successful singleton pregnancies (response rate=93.45\%) (Figure-1). Explanatory variables were maternal demographics, household monthly expenditure and obstetrics history. According to the national guideline on prenatal care, pattern of receiving prenatal care services was categorized into regular (at least 9 visits to healthcare centers), irregular (less than 9 visits) and not receiving. Self-rated health was measured using the following question:" How would you rate your general health status?" on a 5-point scale ranging from 'very good' (a score of 5) to 'very bad'(a score of 1) (12). The Socio-Economic Status (SES) was calculated by the Principal Component Analysis method on ownership of nine household assets. The study outcomes were newborn

sbirthanthropometricmeasures $\in$ clud $\in$ gweight $(\in$ grams $), \leq n>h(\in$ centimeter $)$ and head $\circ$ umference $(\in$ centimeter $)$ and $s$ vaccination card. The exposure of interest was the pattern of water-pipe smoking during the current pregnancy, which was measured by a checklist during the first cohort visit in pregnancy. To detect changes in water pipe smoking, an additional question was asked the subjects:" Did you stop smoking water pipe because you realized you were pregnant?" in the postpartum visit. The pattern of water pipe smoking was then categorized in three distinct groups: 1 ) mothers who never smoke water pipe (never smoker); 2) mothers who stopped water pipe during pregnancy and resumed their smoking behavior postpartum (quitters); 3) mothers who continued water pipe regardless of their pregnancy (always smokers). Second-hand water pipe smoking was also categorized into "yes/no", which was applied for both pregnancy and postpartum period. Bivariate comparisons were performed using Chi-square and t-test. Confounder selection was based on change-in-estimate strategy. Accordingly, covariates for which change in exposure effect estimate (i.e. regression coefficient) falls outside the range of $10 \%$ were considered as the potential confounders (13). Generalized Linear Models (GLMs) with Gaussian family and Identity link function and robust standard errors were utilized to measure the effects of pattern of water pipe smoking on the study outcomes. All the analyses were performed using STATA version 14 (Stata Corp., College Station, TX, USA). For the final model, p-values $<0.05$ were considered as statistically significant.

\section{Results}

Of 1,120 subjects, more than half $(n=583,52 \%)$ were $26-35$ years old and $81 \%(n=916)$ had education levels below high-school diploma. Almost all the subjects belonged to families with four or less members $(n=1,005,89.7 \%)$. More than half $(n=628,56 \%)$ of the subjects reported ownership of less than 4 
assets, and $57 \%(n=639)$ reported a monthly expenditure of less than 41US\$. Overall, 969 subjects (86.5\%) never smoked water pipe during their pregnancy; while 62 subjects (5.5\%), which was equivalent to 58 percent of smokers used water pipe both in pregnancy and postpartum period and 89 subjects (7.9\%), which was equivalent to 42 percent of smokers, quitted water pipe because of pregnancy and resumed it in postpartum. Amongst the always smokers, the mean (SD) age of smoking initiation was $18.4(4.2)$ years and $62 \%$ of the smokers $(n=40)$ had one water pipe session per day. On average, mean birth weight in girls and premature infants (mean birthweight=2943.6 \pm 506 and 2233.3 \pm 780 , respectively) was significantly lower compared to boys and term infants (mean birthweight $=3100.7 \pm 540$, and $3067.3 \pm 465$, respectively; $P<0.0001$ ). More specifically, median and inter-quartile range of birth weight according to the gestational age at delivery was 3000 and 610 grams for term infants and 2220 and 1000 grams for preterm infants. In comparison to poor or average selfrated health, pregnant women with excellent self-rated health had infants with significantly higher mean birthweight (3022 \pm 523 compared to $2978 \pm 603$ in average self-rated health and $3400 \pm 507$ in poor self-rated health, $\mathrm{P}=0.0606$ ). On average, birth length was significantly shorter in families with more than 4 members (48.1 \pm 2.74 vs. $48.6 \pm 2.52 ; \mathrm{P}=0.0006)$. The mean birth length of infants born by Cesarean section delivery was significantly lower than those who were born by vaginal delivery $(48.2 \pm 2.8$ vs. $48.5 \pm 2.4, P=0.0107)$. Furthermore, compared to families with less than 4 members, mean head circumference was significantly lower in families with more than 4 members $(41.07+2.5$ vs. $41.4+2.6, P=0.0070)$. Newborns of never smokers had the highest mean (SD) birth weight $(3035 \pm 527)$ in comparison to newborns of quitters and always smokers $(2914.1 \pm 510$ and $2848.3 \pm 574$, respectively; $P=0.0046)$. The mean (SD) birth length was 48.4(2.6) in never smokers, 47.9 (2.5) in quitters and 48(2.9) in always smokers. Categorization on time of cessation among quitters, we realized that mean (SD) birthweight was 2901.81(102), 2951.29(102), and 2894.94(82) grams in mothers who quitted water pipe in first, second, and third gestational trimester, respectively. The difference of mean birth weight according to the time of cessation did not yield any significant result (P-Value=0.903).

The mean (SD) head circumference was $41.2(2.5)$ in never smokers, 41.3(2.6) in quitters and 41.9(2.5) in always smokers. The difference of mean length and head circumference among the three categories of water pipe smoking did not yield significant results (P-values=0.091 and 0.1007, respectively) (Table-1). The results of the final Generalized Linear Models (GLMs) for birth weight showed that compared to never smokers, quitting water pipe because of pregnancy would decrease mean birthweight by 99.30 grams ( $\beta$ : $-99.30,95 \% \mathrm{Cl}$ : $-204.35,-5.75)$, while sustained smoking behavior during pregnancy will additionally decrease it by 37.83 grams ( $\beta$ : $-137.13 ; 95 \% \mathrm{Cl}:-262.21,-12.05)$. The final model for mean birth length did not yield statistically significant results. Furthermore, after adjustments on important confounders and compared to non-smokers, mean head circumference will increase by 0.79 centimeter in those infants whose mothers were always smokers ( $\beta: 079,95 \% \mathrm{Cl}: 0.13,1.45)($ Table-2).

\section{Discussion}

Exposure to maternal tobacco smoking during pregnancy is a well-recognized cause of fetal growth restriction and preterm birth (14). Tobacco smoking during pregnancy can increase the risk of fetal growth restrictions up to two times, while nearly one-quarter of all infants with growth restriction can be attributed to tobacco smoking $(15,16)$. Regardless of the underlying theory for the effects of smoking cessation on infant health, all the previous studies have focused on cigarette as the mode of smoking. Nevertheless, our study attempted to provide a clearer picture on the effects of water pipe cessation on pregnancy outcomes. In spite of similar health consequences of various types of tobacco smoking, water pipe has been shown to have unique health effects, which could not be captured by studies on cigarette smoking (17). In our large population-based cohort study, we found that $5.5 \%$ of subjects were always smoker; while nearly $8 \%$ of the subjects quitted water pipe following pregnancy notification. Our study showed that continuation of water pipe smoking during pregnancy was associated with a substantial reduction in birth weight. We found that $42 \%$ of water pipe smokers ( $8 \%$ of all study subjects) quit water pipe smoking following pregnancy occurrence. Our study revealed that, the mean birth weight in infants of quitters and always smokers was 99 and 137 grams lighter than mean birth weight in infants of never smokers, respectively. The results we observed are biologically plausible. The effects of maternal tobacco smoking on fetal growth are complex and mainly grounded on two theoretical mechanisms. It may result from an interaction between genetic traits and direct toxic effects of nicotine, or may be due to the placental-smoking interaction. Positive effects of cessation of cigarette smoking on pregnancy outcomes have been reported in few studies $(10,18)$. The results of these investigations; however, have relied heavily on the selected theoretical mechanism. In other words, early cessation of tobacco smoking results in less effect in the former mechanism; whereas a critical window of exposure emerged in the first trimester in the latter one $(18,19)$. Setting aside different concepts, both aforementioned theories imply that the time of cessation might be an important predictor in the pathway of smoking cessation on pregnancy outcomes. Our findings supported the premise that smoking impedes growth and fetal weight gain and reinforced prior conclusions that quitting tobacco smoking will bring about major improvement in fetal growth and birth anthropometries. For instance, Vilalbi et.al reported lower birth weight among mothers who continued cigarette smoking during pregnancy in Spain. The authors concluded that the effects of smoking was most prominent for intra-uterine growth retardation (20). The results of a study on the data from the Generation R Study, a population-based prospective cohort study in the Netherlands, has shown that although pre-conception active cigarette smoking was not associated with adverse pregnancy outcomes; continued active smoking in pregnancy increased the odds of low birthweight by $75 \%$. The authors also reported that quitting smoking in early pregnancy was associated with a higher birthweight than continuing to smoke. (21).

Notwithstanding, the present study has provided a novel piece of information indicating that quitting water pipe smoking during pregnancy might substantially improve fetal growth in water pipe smoking mothers. The estimated effect of quitting water pipe on birth anthropometries remained even after adjusting on preterm birth and second-hand water-pipe smoking, implying the extent to which primary prevention on smoking cessation may be beneficial for all pregnant women. For instance, our data showed that regular prenatal checkups, defined as nine routine checkups throughout pregnancy from healthcare centers, were received by only $35 \%$ of all study subjects. In other words, more than two-thirds of pregnant women in our sample received their prenatal care from private physicians or did not receive it at all. Interestingly, the proportion of women not receiving prenatal care from any sources was significantly higher among always smokers; whereas, never smokers received the care more frequently from healthcare centers (data not shown). This observation has brought an important implication. Encouragement to initiate prenatal healthcare services as soon as possible may assist pregnant women in making the decision to quit water pipe in pregnancy. Such awareness programs should pay special attention to pregnant women from disadvantaged socio-economic households, since our previous work showed that lower socio-economic status was strongly related to less knowledge and attitude about the adverse effects of water pipe smoking (3). In case of cigarette smoking, it has been shown that to achieve the same risk of growth restrictions as non-smoking mothers, quitting must be 
adopted before conception $(9,10)$, although it is still efficient at earlier points during pregnancy $(18)$. Having a similar logic for water pipe smoking, cessation programs should focus on benefits of quitting in preconception period or as early in pregnancy as possible.

Our study was amongst the first attempts to quantify birth anthropometric measures following various patterns of water pipe smoking during pregnancy. Our study used data from a population-based prospective cohort study, which has guaranteed a suitable external generalizability to a wider population of pregnant women in South of Iran. Large sample size of our study $(n=1,120)$ provided satisfactory statistical power to strengthen the precision of our estimates. While previous studies mostly evaluated a single birth outcome such as birthweight, we studied all the three standard anthropometric measures, including weight, length, and head circumference. Nevertheless, our study has suffered from some limitations. In the absence of a ubiquitous valid tool to measure water pipe smoking during pregnancy, we used a self-report checklist to measure the exposure of interest. Therefore, variations in the estimated prevalence of water pipe smokers in our results with others are likely due to measurement biases, mainly reporting bias, stemmed from use of different self-report tools. Moreover, exclusion criteria of the BAPC project excluded pregnant women who had medically assisted conception. Hence, the observed effects of water pipe on birth anthropometries should be interpreted with caution for this subgroup of women.

\section{Conclusions}

Quitting water pipe during pregnancy had positive effects on birth anthropometric measures, especially birth weight. Some suggestions are provided including integration of information ragarding health benefits of quitting smoking into routine prenatal healthcare services and development of awareness programs to encourage smokers to quit early in pregnancy.

\section{Abbreviations}

GLMs: Generalized Linear Models; BAPC: Bandar Abbas Pregnancy Cohort; SES: Socio-Economic Status; SD: Standard Deviation; 95\%Cl: 95\% Confidence Intervals

\section{Declarations}

Ethics approval and consent to participate:

The ethical approval for the present study was granted by National Institute for Medical Research Development (Code:N. IR.NIMAD.REC.1396.205) and by institutional review board of Tehran University of Medical Sciences (Code: 42933244/3246). Verbal informed consent was sought from study subjects prior to interviews.

\section{Consent for publication:}

\section{All the study subjects were assured of confidentiality of their personal information and use of the data only in aggregated} form.

\section{Availability of data and materials:}

The datasets used and/or analyzed during the current study are available from the corresponding author on reasonable request.

\section{Competing interests:}

None.

\section{Funding:}

The funding agency did not have any role in the study design, conduction, analysis and interpretation of the results.

\section{Authors' contributions:}

SN designed the study and finalized the manuscript, $\mathrm{KH}$ supervised the BAPC project and data analysis, AM supervised field work and contributed in data management and analysis, HS and ET contributed to field interviews and data preparation, SL contributed in data analysis and manuscript preparation. All the authors have read and approved the manuscript.

\section{Acknowledgment:}

We would like to forward our gratitude to the National Institute for Medical Research Development for their financial support to conduct the BAPC project. We also wish to thank the BAPC subjects for their cooperation thorough the study.

\section{References}

1. Abraham M, Alramadhan S, Iniguez C, Duijts L, Jaddoe VWV, Den-Dekker HT, et al. (2017). A systematic review of maternal smoking during pregnancy and fetal measurements with meta-analysis. PLoS ONE.12(2):e0170946. doi:10.1371/journal.pone.

2. Tamim H, Yunis KA, H Chemaitelly c, M Alameh d, Nassare A. (2008). Effect of narghile and cigarette smoking on newborn birthweight. BJOG 115:91-7. 
3. Faghir-Ganji M, Asgari E, Jabbari M, Nematollahi S, Hosseini M, Ahmadi-Gharaei $H$, et al. Community health assessment: Knowledge, attitude and practice of women regarding water-pipe smoking in Bandar Abbas. MethodsX. 2019;6:442-6.

4. Mirahmadizadeh A, Nakhaee N. (2008). Prevalence of Waterpipe Smoking among Rural Pregnant Women in Southern Iran. Med Princ Pract.17:435-9.

5. Agha-Molaei T, Zare S. (2008). Cigarette and Hooka using pattern in over-15 population of Bandar Abbas, A population based study. Medical Journal of Hormozgan University.11(4):241-6.

6. Nematollahi S, Mansournia MA, Rahimi-Foroushani A, Mahmoodi M, Alavi A, Shekari M, et al. The effects of water-pipe smoking on birth weight: a population-based prospective cohort study in southern Iran. Epidemiology and Health. 2018;40(ID: e2018008).

7. Aghamolaei T, Eftekhar H, Zare S. Risk factors associated with intrauterine growth retardation(IUGR) in Bandar Abbas. . J Med Sci 2007;7:665-9.

8. Windham GC, Hopkins B, Fenster L, SH. S. Prenatal active or passive tobacco smoke exposure and the risk of preterm delivery or low birth weight. . Epidemiology and Health. 2000;11:427-33.

9. Xaverius PK, O'Reilly Z, Li A, Flick LH, Arnold LD. Smoking Cessation and Pregnancy: Timing of Cessation Reduces or Eliminates the Effect on Low Birth Weight. Maternal and child health journal. 2019;23(10):1434-41.

10. Blatt K, Moore E, Chen A, Van-Hook J, DeFranco EA. Association of Reported Trimester-Specific Smoking Cessation and Fetal Growth Restriction. Obstet Gynecol 2015;125(6):1452-9. doi:10.097/AOG.0000000000000679.

11. Holakouie-Naieni K, Nematollahi S, Mansournia MA, Shekari M, AghaMolayi T, Alavi A, et al. A Population-Based Prospective Study to Identify Contributors of Mother and Child Health in Suburban Communities: The Cohort Profile. Iranian Journal of Public Health. 2018;47(3):441-8.

12. Bruin, A. de, Picavet, H. S. J, Nossikov, Anatoly \& World Health Organization. Regional Office for Europe. (1996). Health interview surveys : towards international harmonization of methods and instruments / edited by A. de Bruin, H. S. J. Picavet \& A. Nossikov. Copenhagen : WHO Regional Office for Europe. https://apps.who.int/iris/handle/10665/107328

13. Greenland S, Pearce N. (2015). Statistical Foundations for Model-Based Adjustments. Annu Rev Public Health.36:89-108.

14. Knopik VS. Maternal smoking during pregnancy and child outcomes: real or spurious effect? . Dev Neuropsychol 2009;34(1):1-36.

15. Mitchell EA, Thompson JM, Robinson E, al. e. Smoking, nicotine and tar and risk of small for gestational age babies. . Acta paediatrica. 2002;91(3):323-8. [PubMed: 12022307].

16. Delpisheh A, Kelly Y, Rizwan S, Attia E, Drammond S, BJ. B. Population attributable risk for adverse pregnancy outcomes related to smoking in adolescents and adults. . Public health 2007;121(11):861-8. [PubMed: 17606278].

17. WHO Study Group on Tobacco Product Regulation (TobReg) (2015). Advisory note: Waterpipe tobacco smoking: health effects, research needs and recommended actions for regulators. 2nd edition. available at: http://www.who.int/tobacco/publications/prod_regulation/waterpipesecondedition/en/

18. Polakowski L, Akinbami L, Mendola P. Prenatal Smoking Cessation and the Risk of Delivering Preterm and Small-for-Gestational-Age Newborns. Obstetrics \& Gynecology. 2009;114(2):318-25.

19. Holloway AC, Salomon A, Soares MJ ea. Characterization of the adverse effects of nicotine on placental development: in vivo and in vitro studies. American journal of physiology Endocrinology and metabolism 2014;306(4):E443-56. [PubMed: 24368670].

20. Villalbi JR, Salvador J, Cano-Serral G, Rodriguez-Sanz MC, C. B. Maternal smoking, social class and outcomes of pregnancy. . Paediatr Perinat Epidemiol 2007;21:441-7.

21. Jaddoe VW, Troe EJ, Hofman A, Mackenbach JP, Moll HA, Steegers EA, et al. Active and passive maternal smoking during pregnancy and the risks of low birthweight and preterm birth: the Generation R Study. Paediatr Perinat Epidemol. 2008;22:162-71.

\section{Table}

Table 1 Newborn`s anthropometric measures at birth by maternal characteristics in the BAPC project 


\begin{tabular}{|c|c|c|c|c|}
\hline Variable & $\mathrm{N}(\%)$ & $\begin{array}{c}\text { Mean Weight } \\
\text { (SD) }\end{array}$ & $\begin{array}{c}\text { Mean Length } \\
\text { (SD) }\end{array}$ & $\begin{array}{c}\text { Mean Head circumference } \\
\text { (SD) }\end{array}$ \\
\hline \multicolumn{5}{|c|}{ Demographic characteristics } \\
\hline \multicolumn{5}{|l|}{ Maternal age } \\
\hline$>15$ & $3(0.27)$ & $2666.66(351.18)$ & $47.33(2.08)$ & $40.97(0.19)$ \\
\hline $16-25$ & $439(39.20)$ & $3033.03(499.29)$ & $48.50(2.48)$ & $41.31(2.67)$ \\
\hline $26-35$ & $583(52.05)$ & $3015.00(551.12)$ & $48.30(2.84)$ & $41.26(2.48)$ \\
\hline$<35$ & $95(8.48)$ & $3040.42(537.74)$ & $48.71(1.95)$ & $41.36(2.70)$ \\
\hline \multicolumn{5}{|c|}{ Maternal education } \\
\hline illiterate & $51(4.55)$ & $3121.96(704.28)$ & $48.29(2.80)$ & $41.37(2.70)$ \\
\hline Below diploma & $916(81.79)$ & $3016.72(531.46)$ & $48.38(2.61)$ & $41.29(2.59)$ \\
\hline academic & $153(13.66)$ & $3029.73(444.95)$ & $48.66(2.72)$ & $41.23(2.40)$ \\
\hline \multicolumn{5}{|l|}{ Maternal job } \\
\hline With income & $28(2.5)$ & $2987.14(405.61)$ & $48.53(2.45)$ & $40.86(2.90)$ \\
\hline Without income & $1092(97.50)$ & $3024.22(532.53)$ & $48.41(2.64)$ & $41.30(2.56)$ \\
\hline \multicolumn{5}{|l|}{ SES (Item) } \\
\hline$>4$ & $628(56.07)$ & $3028.14(538.14)$ & $48.64(2.52)$ & $41.46(2.60)$ \\
\hline$\leq 4$ & $492(43.93)$ & $3017.10(519.03)$ & $48.12(2.74)$ & $41.07(2.52)$ \\
\hline \multicolumn{5}{|c|}{ Household Monthly expenditure (US\$) } \\
\hline$\leq 41.00$ & $639(57.05)$ & $3026.04(522.58)$ & $48.24(2.63)$ & $41.30(2.61)$ \\
\hline $41.00-83.99$ & $220(19.64)$ & $2992.59(514.07)$ & $48.64(2.50)$ & $41.48(2.51)$ \\
\hline $84.00-166.99$ & $215(19.20)$ & $3044.81(577.72)$ & $48.63(2.73)$ & $41.07(2.47)$ \\
\hline$>167.00$ & $46(4.11)$ & $3031.30 \quad(472.18)$ & $48.79(2.69)$ & $41.18(2.73)$ \\
\hline \multicolumn{5}{|l|}{ Family size } \\
\hline$\leq 4$ & $1005(89.73)$ & $3017.02(537.95)$ & $48.60(2.52)$ & $41.40(2.58)$ \\
\hline$>4$ & $115(10.27)$ & $3078.08(448.68)$ & $48.10(2.74)^{*}$ & $41.07(2.53)^{*}$ \\
\hline \multicolumn{5}{|c|}{ Pregnancy characteristics } \\
\hline \multicolumn{5}{|c|}{ Receiving prenatal care from Healthcare centers } \\
\hline Regular & $391(34.91)$ & $3028.63(524.22)$ & $48.47(2.36)$ & $41.19(2.53)$ \\
\hline Irregular & $717(64)$ & $3018.02(534.38)$ & $48.37(2.79)$ & $41.34(2.60)$ \\
\hline Not received & $12(1.07)$ & $3164.16(423.24)$ & $48.70(1.25)$ & $41.09(1.77)$ \\
\hline \multicolumn{5}{|c|}{ Maternal Self-rated health } \\
\hline Poor/very poor & $10(0.89)$ & $3400.00 \quad(507.71)$ & $47.95(3.93)$ & $40.52(2.80)$ \\
\hline Medium & $77(6.88)$ & $2978.83(603.31)$ & $48.27(2.67)$ & $41.26(2.43)$ \\
\hline $\begin{array}{l}\text { good } \\
\text { /excellent }\end{array}$ & $1033(92.23)$ & $3022.96(523.04)^{*}$ & $48.43(2.62)$ & $41.30(2.58)$ \\
\hline \multicolumn{5}{|c|}{ Anemia during pregnancy } \\
\hline Yes & $311(27.77)$ & $3020.87(487.68)$ & $48.46(2.37)$ & $41.29(2.46)$ \\
\hline No & $809(72.23)$ & $3024.22(545.17)$ & $48.39(2.73)$ & $41.29(2.61)$ \\
\hline \multicolumn{5}{|l|}{ Delivery type } \\
\hline Vaginal & $647(57.77)$ & $3038.44(472.93)$ & $48.57(2.48)$ & $41.34(2.61)$ \\
\hline Cesarean & $473(42.23)$ & $3002.56(598.45)$ & $48.20(2.82)^{*}$ & $41.22(2.52)$ \\
\hline \multicolumn{5}{|l|}{ Infant sex } \\
\hline Boy & $568(50.71)$ & $3100.70 \quad(540.63)$ & $48.63(2.76)$ & $41.47(2.68)$ \\
\hline Girl & $552(49.29)$ & $2943.64(506.32)^{*}$ & $48.18(2.48)$ & $41.10(2.44)$ \\
\hline \multicolumn{5}{|l|}{ Preterm delivery } \\
\hline Yes & $70(6.25)$ & $2233.35(780.19)$ & $45.37(4.81)$ & $41.12(3.26)$ \\
\hline No & $1050(93.75)$ & $3067.34(465.62)^{*}$ & $48.62(2.28)$ & $41.30(2.52)$ \\
\hline \multicolumn{5}{|c|}{ Water pipe smoking } \\
\hline
\end{tabular}




\begin{tabular}{|l|c|cc|c|c|} 
Never smoker & $969(86.52)$ & 3035.18 & $(527.08)$ & $48.48(2.62)$ & $41.24(2.57)$ \\
\hline Quitter & $89(7.95)$ & 2914.12 & $(510.89)$ & $47.96(2.51)$ & $41.35(2.64)$ \\
\hline Always Smoker & $62(5.54)$ & 2848.30 & $(574.78)$ & $48(2.90)$ & $41.96(2.50)$ \\
\hline \multicolumn{5}{|l|}{ Second-hand tobacco smoking } \\
\hline Yes & $183(16.4)$ & $3042.3(502.8)$ & $48.3(2.85)$ & $41.27(2.35)$ \\
\hline No & $937(83.6)$ & $3009.9(535.9)$ & $48.4(2.59)$ & $41.29(2.61)$ \\
\hline
\end{tabular}

Table 2- Adjusted effects of quitting water pipe smoking on infant`s anthropometric measures

\begin{tabular}{l|c|c|c|c|c|c|c|c|c|}
\hline \multirow{2}{*}{ er pipe pattern } & \multicolumn{3}{|c|}{ Birthweight $^{\Omega}$} & \multicolumn{3}{c|}{ Birth length $\psi$} & \multicolumn{3}{c|}{ Birth head circumference } \\
\cline { 2 - 9 } & \multicolumn{2}{|c|}{$95 \%$ CI } & P & $\beta$ & $95 \%$ CI & P & $\beta$ & $95 \%$ CI & P \\
\hline - smoker & Reference & & & & & & & \\
\hline
\end{tabular}

$\Omega$ : adjusted for: self-rated health, infant sex, family size, SES, preterm delivery, secondhand water pipe smoking $\psi$ adjusted for: infant sex, SES, delivery type, secondhand water pipe smoking $€$ adjusted for: infant sex, SES, preterm delivery, secondhand water pipe smoking

\section{Figures}

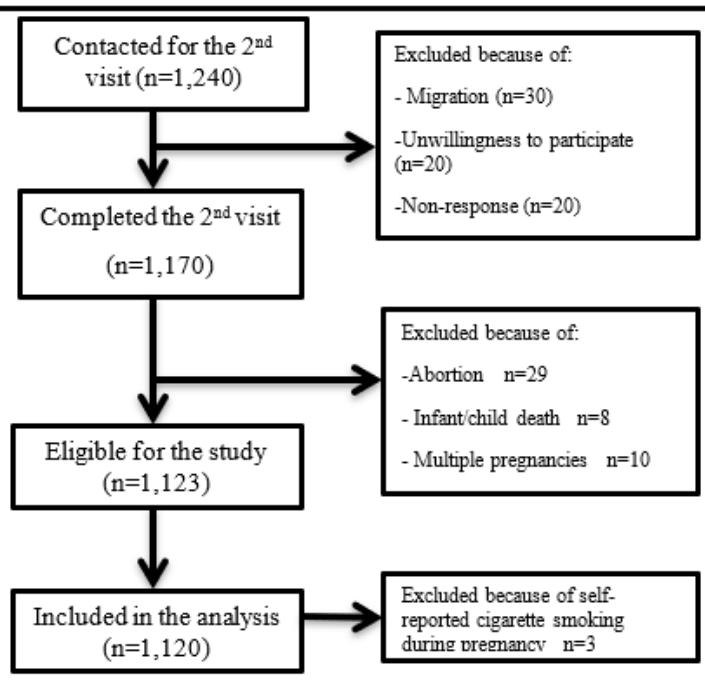

Figure 1

Flowchart of the BAPC subjects participated in the present study 